Erratum

\title{
Differential Analysis of Hypertension-Associated Intestinal Microbiota
}

\author{
Xie Dan ${ }^{1}$, Zhang Mushi ${ }^{1}$, Wang Baili², Lin Han ${ }^{1}$, Wu Enqi ${ }^{1}$, Zhao Huanhu ${ }^{1}$, Li Shuchun ${ }^{1,}{ }^{凶}$ \\ 1. School of Pharmacy, Minzu University of China, Key Laboratory of Ethnomedicine (Minzu University of China), Ministry of education, Beijing 100081, P.R. \\ China \\ 2. School Hospital, Minzu University of China, Beijing 100081, China \\ $\triangle$ Corresponding author: ShuChun Li, MinZu University of China, 27 ZhongGuanCun South Street, 100081, Beijing, China. Tel: 86-10-6893-3254; Fax: \\ 86-10-6893-9905; E-mail: jason@muc.edu.cn
}

(C) The author(s). This is an open access article distributed under the terms of the Creative Commons Attribution License (https://creativecommons.org/licenses/by/4.0/). See http://ivyspring.com/terms for full terms and conditions.

Published: 2021.10 .08

Corrected article: Int J Med Sci 2019; 16(6): 872-881. doi: 10.7150/ijms.29322.

In our paper [1], the first sentence of the result should be corrected as "A total of 67 cases of HTN and 62 cases of normal BP were included in this study", and Table 1 should be corrected as follows.

Table 1. Characteristics of subjects

\begin{tabular}{|c|c|c|c|}
\hline & $\begin{array}{l}\text { Normal } \\
\text { pressure }\end{array}$ & $\begin{array}{l}\text { Blood } \\
\text { Hypertension }\end{array}$ & P-value \\
\hline Sample & 62 & 67 & \\
\hline Gender (\% Female) & $62(53.23 \%)$ & $67(61.19 \%)$ & 0.360 \\
\hline Age, year & $69.322 \pm 10.613$ & $69.492 \pm 9.630$ & 0.509 \\
\hline $\mathrm{SBP}, \mathrm{mmHg}$ & $122.935 \pm 6.902$ & $153.298 \pm 14.917$ & $0.000^{*}$ \\
\hline $\mathrm{DBP}, \mathrm{mmHg}$ & $76.209 \pm 6.902$ & $84.313 \pm 10.739$ & $0.000^{*}$ \\
\hline BMI, $\mathrm{kg} / \mathrm{m}^{2}$ & $26.089 \pm 3.112$ & $25.051 \pm 4.436$ & 0.169 \\
\hline WHR & $0.864 \pm 0.065$ & $0.853 \pm 0.130$ & 0.645 \\
\hline GLU, mmol/L & $6.729 \pm 1.956$ & $6.030 \pm 1.176$ & 0.059 \\
\hline $\mathrm{CHO}, \mathrm{mmol} / \mathrm{L}$ & $5.249 \pm 1.022$ & $5.093 \pm 1.062$ & 0.443 \\
\hline $\mathrm{HDL}, \mathrm{mmol} / \mathrm{L}$ & $1.403 \pm 50.827$ & $1.441 \pm 0.346$ & 0.496 \\
\hline $\mathrm{LDL}, \mathrm{mmol} / \mathrm{L}$ & $1.986 \pm 1.250$ & $3.182 \pm 0.865$ & 0.322 \\
\hline $\mathrm{TG}, \mathrm{mmol} / \mathrm{L}$ & $1.986 \pm 1.250$ & $1.812 \pm 1.206$ & 0.336 \\
\hline BUN, mmol/L & $5.373 \pm 1.285$ & $5.739 \pm 1.418$ & 0.181 \\
\hline $\mathrm{UA}, \mu \mathrm{mol} / \mathrm{L}$ & $344.709 \pm 89.964$ & $346.224 \pm 76.830$ & 0.386 \\
\hline $\mathrm{HCY}, \mu \mathrm{mol} / \mathrm{L}$ & $13.940 \pm 6.080$ & $13.150 \pm 3.620$ & 0.607 \\
\hline
\end{tabular}

The data on age, SBP, DBP, BMI, WHR, GLU, CHO, HDL, LDL, TG, BUN, UA, and HCY are expressed as the mean \pm SD. P-values for gender, age, SBP, DBP, BMI, WHR, GLU, CHO, HDL, LDL, TG, BUN, UA, and HCY were calculated using Student's $t$-test. ${ }^{*} \mathrm{P}<0.05$. SBP: systolic blood pressure, DBP: diastolic blood pressure, BMI: body mass index, WHR: waist-to-hip ratio; GLU: glucosuria; HDL: high density lipoprotein; LDL: low density lipoprotein; TG: total triglyceride, CHO: total cholesterol; BUN blood urea nitrogen; UA: uric acid; HCY: homocysteine. ${ }^{*} \mathrm{P}<0.05$.

\section{References}

1. Dan X, Mushi Z, Baili W, Han L, Enqi W, Huanhu Z, Shuchun L. Differential Analysis of Hypertension-Associated Intestinal Microbiota. Int J Med Sci. 2019; 16(6):872-881 doi: $10.7150 /$ ijms. 29322 